Department of

Cardiology, Istituto

Clinico Humanitas, 56

Rozzano, 20089 Milan,

Italy

M Gasparini

M Mantica

P Turco

Department of

Cardiology, Ospedali

Riuniti, Lavagna, Italy

M Brignole

L Gianfranchi

Department of

Cardiology, Ospedale S

Maria Nuova, Reggio

Emilia, Italy

C Menozzi

Department of

Cardiology, Santo

Spirito Hospital,

Casale Monferrato,

Italy

F Pizzetti

"De Gasperis"

Department of

Cardiology Niguarda

Hospital, Milan, Italy

G Magenta

Department of

Cardiology, Ospedale

Feltre, Belluno, Italy

P Delise

Department of

Cardiology, Udine

Hospital, Italy

A Proclemer

Department of

Cardiology, Piombino

Hospital, Italy

$S$ Tognarin

Department of

Cardiology, Vicenza

Hospital, Italy

R Ometto

Department of Cardiology, Ospedale di Circolo Varese, Italy

F Acquati

Department of

Cardiology,

Pontesampiero

Hospital, Italy

R Mantovan

Department of

Cardiology, IRCCS

Policlinico S Matteo

Pavia, Italy

G M De Ferrari

Correspondence to:

Dr Gasparini.

email: maurizio.gasparini@

humanitas.it

Accepted for publication 12 April 1999

\title{
Thromboembolism after atrioventricular node ablation and pacing: long term follow up
}

\author{
M Gasparini, M Mantica, M Brignole, L Gianfranchi, C Menozzi, F Pizzetti, G Magenta, \\ P Delise, A Proclemer, S Tognarin, R Ometto, F Acquati, R Mantovan, P Turco, \\ G M De Ferrari
}

\begin{abstract}
Objective-To assess the incidence of arterial embolic events in patients with high rate, drug resistant, severely symptomatic paroxysmal and chronic atrial fibrillation who have undergone atrioventricular (AV) node ablation and permanent pacing.
\end{abstract}

Design-Multicentre retrospective cohort study.

Patients and management-From May 1987 to January 1997, AV node ablation was performed in 585 severely symptomatic patients (mean (SD) age 66 (11) years) with high rate, drug resistant paroxysmal atrial fibrillation (308) or chronic atrial fibrillation (277). Lone atrial fibrillation was present in 133 patients, while the remaining 452 suffered from dilated, ischaemic, or valvar heart disease. Patients underwent VVIR (454) or DDDR (131) pacemaker implantation, after AV node ablation. Antiplatelet agents were given to 202 patients, warfarin to 187 patients.

Results-During a follow up of 33.6 (24.2) months, thromboembolic events were observed in 17 patients (3\%); the actuarial occurrence rates of thromboembolism were $1.1 \%, 3 \%, 4.2 \%$, and $7.4 \%$ after one, three, five, and seven years, respectively. Among five variables, univariate analysis showed that only the presence of chronic atrial fibrillation at the time of ablation (relative risk $(R R)=1.8,95 \%$ confidence interval $(\mathrm{CI})=1.02$ to $3.20, \mathrm{p}=0.04)$ and the need for warfarin treatment $(\mathrm{RR}=1.6,95 \%$ CI 1.00 to $2.71, \mathrm{p}=0.048)$ were associated with a significantly higher risk of occurrence of thromboembolic events. On multivariate analysis the only predictor of embolic events during the follow up was the presence of chronic atrial fibrillation.

Conclusions-Data from this large cohort of patients indicate a fairly low incidence $\mathbf{1 . 0 4 \%}$ per year) of thromboembolic events after AV node ablation and pacing for drug refractory, high rate atrial fibrillation.

(Heart 1999;82:494-498)

Keywords: atrial fibrillation; embolism, atrioventricular node ablation; pacemaker implantation

Atrial fibrillation is one of the most common arrhythmias observed in clinical practice. Embolic stroke is a frequent complication of atrial fibrillation and results in death or severe neurological damage in $50-70 \%$ of the affected patients. ${ }^{1}$

The risk of systemic embolic events is well documented in both paroxysmal and chronic atrial fibrillation, ranging from less than $1 \%$ per year in lone paroxysmal atrial fibrillation ${ }^{2}$ to more than $7 \%$ per year in patients with chronic atrial fibrillation and additional cardiac or non-cardiac risk factors for embolic events. ${ }^{34}$ Thus atrial fibrillation is the cardiovascular disease most often associated with systemic embolism. ${ }^{5}$

Despite significant advances in the pharmacological approach to patients with atrial fibrillation, systemic embolism is still a major cause of death and disability. In a meta-analysis of five randomised trials evaluating the best pharmacological methods of preventing embolism among patients with atrial fibrillation, ${ }^{6}$ the combined incidence of stroke and systemic embolism was $1.7 \%$ per year among warfarin treated patients and 5\% per year in the control group. Notably, warfarin treated patients had an incidence of major haemorrhage of $1.3 \%$ per year.

A non-pharmacological approach consisting of radiofrequency atrioventricular (AV) node ablation followed by pacemaker implantation has recently become a reliable alternative treatment in patients with high rate, drug resistant atrial fibrillation. Catheter ablation of the AV junction followed by pacing can significantly reduce symptoms and improve the quality of life of patients with drug refractory atrial fibrillation. ${ }^{78}$ The persistence of atrial fibrillation could leave the patient at risk of some of the potentially deleterious consequences of this condition, specifically of systemic embolism. ${ }^{9}$ However, the risk of embolic events among patients treated with AV node ablation and pacing has never been properly evaluated. Rosenqvist et al anecdotally observed the absence of systemic embolism among 29 patients with paroxysmal atrial fibrillation or chronic atrial fibrillation treated with direct current ablation of the AV node. ${ }^{7}$ They suggested that rate control may have reduced the development of cardiac thrombi.

In the multicentre study reported here, we assessed the incidence of arterial embolic events in patients with high rate, drug resistant, severely symptomatic paroxysmal atrial fibrillation and chronic atrial fibrillation who had undergone AV node ablation and permanent pacing. 
Table 1 Clinical data

\begin{tabular}{ll}
\hline & $n(\%)$ \\
\hline Number of patients & 585 \\
Age (years) & $66(11)$ \\
$\quad$ mean (SD) & $68(21$ to 90$)$ \\
median (range) & \\
NYHA class & $2.3(1)$ \\
mean (SD) & $2(1$ to 4$)$ \\
median (range) & $3.5(1.7)$ \\
Ineffective AA drugs & $3(1$ to 9$)$ \\
mean (SD) & $26(4)$ \\
median (range) & $168(29)$ \\
Previous embolic events & $85(15)$ \\
No heart disease & $86(15)$ \\
Hypertension & $131(22)$ \\
Ischaemic heart disease & $82(14)$ \\
Dilated cardiomyopathy & $33(6)$ \\
Valvar heart disease & $277(47)$ \\
Prosthetic valve & $308(53)$ \\
Chronic AF & \\
Paroxysmal AF & \\
\hline
\end{tabular}

AA, antiarrhythmic; AF, atrial fibrillation; NYHA, New York Heart Association functional class.

\section{Methods}

STUDY POPULATION

The study population consisted of 585 patients (mean (SD) age 66 (11) years) with high rate, drug resistant, severely symptomatic atrial fibrillation who had undergone direct current or radiofrequency ablation of the AV node. In this population there was an important incidence of heart disease $(71 \%$ of the population, and in particular, $42 \%$ of patients presented dilated cardiomyopathy, valvar heart disease, or a prosthetic valve): these patients underwent AV node ablation only when every pharmacological approach had failed to control severe symptoms or signs of heart failure (mean 3.5 ineffective antiarrhythmic drugs). The main clinical characteristics of this patient population are given in table 1 .

The procedures were carried out between May 1987 and January 1997 in 12 cardiology departments which perform invasive electrophysiology. This population was derived from a total of 601 patients who underwent AV node ablation during this period: in 16 patients no reliable information about the potential occurrence of embolism could be obtained and these were excluded from the analysis.

AV NODE ABLATION AND PACEMAKER

IMPLANTATION

After informed consent had been given, patients underwent catheter ablation of the AV junction. In 90 patients catheter ablation with direct current energy was performed, according to the technique described by Clementy, ${ }^{10}$ while in the remaining 495 patients radiofrequency (RF) energy was used, as described by

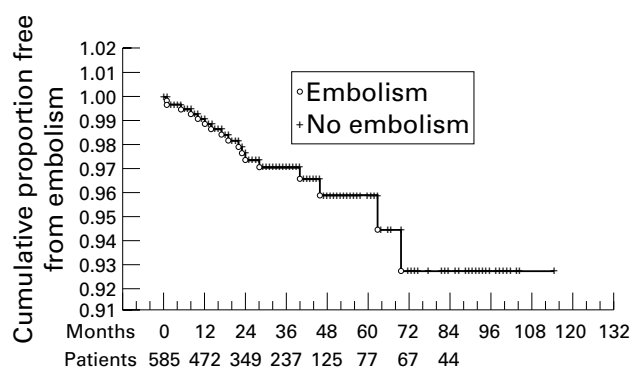

Figure 1 Cumulative proportion freedom from embolic events curve (Kaplan-Meier).
Jackman et al. ${ }^{11}$ After AV node ablation, a permanent pacemaker was implanted in all patients one to 24 hours after ablation: a DDD/DDDR model was used in 131 patients $(22 \%)$ and a VVI/VVIR model in 454 patients (78\%).

POSTABLATION MANAGEMENT

The choice of the drug treatment to prevent embolism was left to the attending physicians, who made the decision on the basis of the clinical condition of the patient and their own preferences. Overall, 187 patients (32\%) were treated with oral anticoagulants, maintaining the international normalised ratio (INR) between 2 and $4 ; 202$ patients $(35 \%)$ were treated with aspirin (from 100 to $325 \mathrm{mg} /$ day); and 196 patients (33\%) did not receive anticoagulant or antiplatelet treatment.

\section{CLINICAL FOLLOW UP}

Follow up data with respect to arterial embolic events and deaths were obtained by retrieving patients' files and, when necessary, by a visit or a telephone contact. An arterial embolic event was defined as the occurrence of a transient ischaemic attack, stroke, or peripheral arterial embolism.

\section{STATISTICAL ANALYSIS}

The data were first subjected to traditional descriptive analysis and are reported in the text as mean (SD), and median values for continuous variables. Comparison was performed by means of the $\chi^{2}$ test for categorical variables and by the two tailed unpaired Student's $t$ test for continuous, normally distributed variables. Actuarial curves for freedom from embolic events were calculated using the Kaplan-Meier method. Comparison between the event-free survival curves for different groups was performed by the log-rank test. Univariate and multivariate predictors of event-free survival were evaluated using a Cox proportional hazard model. A probability $(\mathrm{p})$ value $<0.05$ was considered significant. All $p$ values reported are two tailed.

\section{Results}

ABLATION SUCCESS RATE

Stable complete heart block was achieved in 578 of 585 patients $(98 \%)$ after a mean of 4.8 (5.3) (median 3) radiofrequency pulses (or 2.8 (1.7) shocks in those undergoing the direct current shock approach). In 27 cases (4.6\%) left sided catheterisation was necessary, following repeated unsuccessful application of the conventional right sided approach. Procedure related complications were observed in $2.7 \%$ of cases (four cases of sustained ventricular tachycardia or fibrillation; three peripheral arterial emboli; two pericardial effusions; three local haemorrhages; five deep vein thromboses; one fatal pulmonary embolism secondary to deep vein thrombosis).

\section{FOLLOW UP}

Patients were followed up for an average of 33.6 (24.2) months (median 29 months). During that period, a total of 17 arterial 
Table 2 Comparison of the occurrence of embolism on the basis of different patient characteristics

\begin{tabular}{|c|c|c|c|}
\hline & Emboli & $\%$ & $p$ Value * \\
\hline \multicolumn{4}{|l|}{ Age (years) } \\
\hline$<65$ & $4 / 222$ & 1.8 & \\
\hline$>65$ & $13 / 363$ & 3.6 & NS \\
\hline \multicolumn{4}{|l|}{ NYHA class } \\
\hline I & $3 / 122$ & 2.4 & \\
\hline II & $5 / 171$ & 2.9 & \\
\hline III & $7 / 167$ & 4.2 & \\
\hline IV & $1 / 69$ & 1.4 & NS \\
\hline \multicolumn{4}{|l|}{ Type of pacemaker } \\
\hline VVIR & $14 / 454$ & 3 & \\
\hline DDDR & $3 / 131$ & 2.3 & NS \\
\hline \multicolumn{4}{|c|}{ Presence of heart disease } \\
\hline Lone AF & $2 / 168$ & 1.2 & \\
\hline Heart disease & $15 / 417$ & 3.6 & NS \\
\hline \multicolumn{4}{|l|}{ Treatment } \\
\hline No drug & $1 / 196$ & 0.5 & \\
\hline Antiplatelet agents & $6 / 202$ & 3 & \\
\hline Warfarin & $10 / 187$ & 5.3 & 0.02 \\
\hline \multicolumn{4}{|l|}{ Atrial fibrillation } \\
\hline Paroxysmal & $5 / 277$ & 1.8 & \\
\hline Chronic & $12 / 308$ & 3.2 & 0.05 \\
\hline \multicolumn{4}{|c|}{ Previous embolic events } \\
\hline Yes & $2 / 26$ & 7.7 & \\
\hline No & $15 / 259$ & 2.7 & NS \\
\hline
\end{tabular}

${ }^{\star} \chi^{2}$ test.

$\mathrm{AF}$, atrial fibrillation; NYHA, New York Heart Association functional class.

thromboembolic events (3\%) occurred, including the three periprocedural arterial embolic events: 10 non-fatal and one fatal stroke, five transient ischaemic attacks, and one peripheral arterial embolism. The global actuarial curve for freedom from embolic events calculated using the Kaplan-Meier method is shown in fig 1 . After a mean follow up of one year, embolism-free survival was 98.9\% (95\% confidence interval (CI) $98.0 \%$ to $99.8 \%$ ), at three years it was $97.0 \%(95.4 \%$ to $98.6 \%)$, at five years, $95.8 \%(93.5 \%$ to $98.1 \%)$, and at seven years, $92.6 \%(87.7 \%$ to $97.5 \%)$.

Comparison of the overall frequency of embolism on the basis of different clinical characteristics is shown in table 2. Figure 2 shows the comparison between embolism-free survival curves by the log-rank test for paroxysmal atrial fibrillation $v$ chronic atrial fibrillation. A significant difference was found between these curves $(p=0.03)$. The two survival curves diverge more widely after the first 48 months of follow up, a point reached, however, by only $20.0 \%$ of the patients enrolled. A difference was also found on the basis of antithrombotic treatment $(\mathrm{p}=0.01)$, with patients on warfarin showing the worst embolism-free survival and patients with no

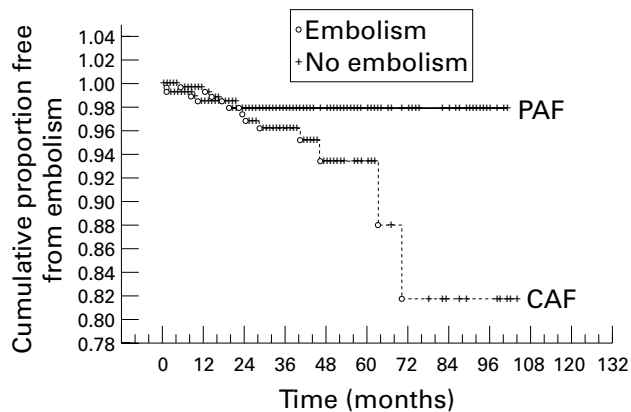

Figure 2 Cumulative proportion freedom from embolic event curves (log-rank test): comparison between patients with paroxysmal atrial fibrillation (PAF) and chronic atrial fibrillation $(C A F)$.

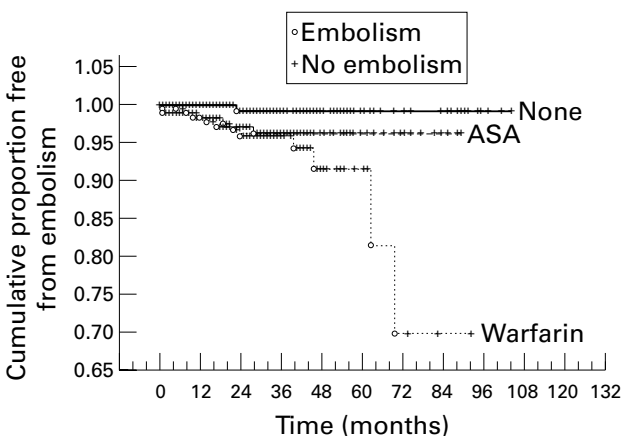

Figure 3 Cumulative proportion freedom from embolic events curves (log-rank test): comparison between patients with no antithrombotic treatment (none), aspirin (ASA), and warfarin.

treatment the best survival (fig 3). The Cox prediction model for event-free survival identifies as univariate predictors of embolism the type of arrhythmia (chronic atrial fibrillation $v$ paroxysmal atrial fibrillation) (relative risk $(R R)=1.8,95 \%$ CI 1.02 to $3.20, p=0.04)$, and the need for warfarin treatment $(R R=1.6$, $95 \%$ CI 1.00 to $2.71, \mathrm{p}=0.048$ ), whereas the presence of heart disease ( $\mathrm{RR}=1.9,95 \% \mathrm{CI}$ 0.9 to $3.9, \quad \mathrm{p}=0.08)$, age $>65$ years $(\mathrm{RR}=1.6,95 \%$ CI 0.9 to $2.9, \mathrm{p}=0.08)$, and NYHA class > I $(R R=0.9,95 \%$ CI 0.5 to 1.7 , $\mathrm{p}=0.75)$ did not provide significant predictive value.

Multivariate analysis showed that chronic atrial fibrillation was the only significant independent predictor of embolism-free survival $(R R=1.8,95 \%$ CI 1.02 to 3.20 , $\mathrm{p}=0.04)$.

Eighty deaths were recorded (13.7\%): 39 non-cardiac, one fatal stroke, 23 non-sudden cardiac, and 17 sudden cardiac. The mortality for the overall population was $4.88 \%$ per year. The actuarial survival curve was $94 \%$ (95\% CI $92 \%$ to $96 \%)$ at one year, $90 \%(95 \%$ CI $87 \%$ to $93 \%$ ) at two years, $85 \%$ (95\% CI $82 \%$ to $88 \%)$ at three years, $79 \%(95 \%$ CI $74 \%$ to $84 \%)$ at five years, and $76 \%$ (95\% CI $68 \%$ to $84 \%$ ) at seven years.

\section{Discussion}

Atrial fibrillation is associated with a twofold increase in all cause mortality ${ }^{12}$; it is present in $6-24 \%$ of patients who have an ischaemic stroke, and in up to $50 \%$ of patients with cardioembolic strokes. ${ }^{13}{ }^{14}$ Compared with the general population, patients with atrial fibrillation and rheumatic valve disease have a 17 -fold increase in risk of embolism, while patients with non-rheumatic atrial fibrillation have a fivefold increase in risk. ${ }^{12}$ The meta-analysis of the pooled data of five large clinical trials on the effects of anticoagulation in patients with atrial fibrillation identified, by multivariate analysis, four independent clinical features that were associated with an increased risk of stroke among patients with no underlying heart disease. These were: a previous transient ischaemic attack, diabetes mellitus, hypertension, and increasing age. ${ }^{6}$ Patients with none of these risk factors showed an incidence of embolism of $1 \%$ per year, whereas patients 
older than 75 years with one or more risk factor showed an incidence of embolism as high as $8 \%$ per year. $^{6}$

Catheter ablation of the AV junction followed by pacing can significantly reduce symptoms and improve the quality of life of patients with drug refractory atrial fibrillation. ${ }^{78}$ This beneficial effect is obtained by means of adequate and regular ventricular rate, in the absence of any demonstrated effect on the atrial arrhythmic substrate. The persistence of atrial fibrillation may conceivably leave the patient at risk of some of the potentially deleterious consequences of this condition, and specifically of systemic embolism. ${ }^{9}$

While a wealth of information regarding the embolic risk is available on patients with atrial fibrillation treated with conventional pharmacological agents, data concerning patients with atrial fibrillation treated with AV node ablation and pacemaker implantation are extremely limited. Indeed, the majority of the published studies report only the occurrence of fatal embolic events in a limited number of patients. ${ }^{75-17}$ However, we recently found a low incidence of embolism in a group of 132 patients treated with AV junction ablation in comparison with a group of 89 patients treated by a conventional pharmacological approach. ${ }^{18}$

Our study is the first to report long term follow up data on a large cohort of patients with AV node ablation (1608 patient-years), specifically investigated to assess the incidence of arterial embolism after the ablation procedure. Even if the incidence of some embolic events, such as transient ischaemic attacks, could have been underestimated owing to the retrospective design of the study, nonetheless our data suggest a low overall incidence of embolic events during follow up (about 1\% per year), which compares very favourably with the incidence observed in similar populations of patients in the major studies. ${ }^{19-23}$ For instance, in the patients enrolled in the stroke prevention in atrial fibrillation (SPAF) study, ${ }^{21}$ who had a similar mean age (67 years) but a lower incidence of relevant heart disease compared with our population, the average incidence of stroke was $2.3 \%$ per year among patients treated with warfarin, $3.6 \%$ per year among those treated with aspirin, and $6.3 \%$ per year among those given placebo. Thus it was higher than observed in the present study, despite the fact that patients with valvar heart disease ( $20 \%$ of our patients), who are known to be at greatest risk of embolism, were not enrolled in the SPAF, and that only definite stroke-not transient ischaemic attacks or peripheral emboli-was considered as an end point.

Given the very limited number of embolic events in our population, the power to assess the predictive value of clinical variables was limited. However, we observed a significantly increased risk of embolism among patients with chronic atrial fibrillation in comparison with paroxysmal atrial fibrillation. The difference increased progressively with time and was particularly evident after more than three years of follow up.
On univariate analysis, in addition to the type of arrhythmia, the type of antithrombotic treatment was also significantly associated with embolism-free survival. Patients on warfarin had a higher incidence of embolism than untreated patients and those on antiplatelet treatment alone. This finding may appear surprising, given the demonstrated efficacy of anticoagulation in the prevention of embolism. However, it should be remembered that the choice of treatment in this study was not randomised and was left to the attending physician. Thus it is possible that the overall clinical judgement of the physician who chose the treatment for the individual patient (probably choosing warfarin for the patients considered to be at greater embolic risk) was superior to the estimate derived from the analysis of a few categorical clinical variables (such as the type of arrhythmia, presence of underlying heart disease, and so on) in predicting the risk of embolic events. This hypothesis appears to be confirmed by the finding that the greatest incidence of embolism (9\%) was observed in patients with prosthetic valves, who were all treated with warfarin.

The fairly low rate of thromboembolic events observed raises the question as to whether ablation may have some protective action against thromboembolism. Rosenqvist et al suggested that rate control may be an important factor in preventing the development of cardiac thrombi. ${ }^{7}$ Indeed, a lower and more regular heart rate, as is achieved by pacing following AV node ablation, can prevent the decrease in cardiac output related to high rate atrial fibrillation which may favour intracardiac thrombi. Moreover, Rodriguez et al showed a significant reduction in the size of the left atrium after AV node ablation, particularly in patients with an ejection fraction less than $50 \%{ }^{24}$ This decrease in left atrial size could play a significant role in the low incidence of embolism after AV node ablation. These hypotheses seem to be in agreement with the finding that both left ventricular dysfunction and the size of the left atrium are significant predictors of thromboembolism. ${ }^{25}$

The incidence of systemic embolism after AV node ablation was particularly low among patients with paroxysmal atrial fibrillation. Almost half of these patients had received a dual chamber pacemaker. Among patients with sick sinus syndrome and a history of paroxysmal atrial fibrillation, atrial pacing-compared with ventricular pacing alone-reduces the incidence of chronic atrial fibrillation and thromboembolic complications, as well as left atrial size. ${ }^{26} 27$ Thus it may be speculated that part of the beneficial effects observed in our study among patients with paroxysmal atrial fibrillation might have resulted from prevention of sustained episodes of fibrillation by atrial pacing.

\section{LIMITATIONS OF THE STUDY}

The major limitations of this multicentre study are the absence of a control group, which makes a precise estimate of the beneficial effects provided by AV node ablation and pac- 
ing impossible, and its retrospective design. However, our population consisted of patients with an important incidence of heart disease. These patients only underwent AV node ablation when every pharmacological approach had failed to control severe symptoms or signs of heart failure; indeed, assigning patients with these clinical characteristics to a noninterventional approach could be subjected to criticism, given the already proven inefficacy of any pharmacological treatment in this particular population. Thus future attempts to estimate the beneficial effects on systemic embolism provided by AV node ablation and pacing compared with the traditional treatment will have to involve prospective studies that enrol and randomise patients in a less advanced phase of the disease.

1 Cairns JA, Connolly SJ. Nonrheumatic atrial fibrillation: risk of stroke and role of antithrombotic therapy. Circulation $1991 ; 84: 469-81$

2 Morley J, Marinchak R, Rials ST, et al. Atrial fibrillation, anticoagulation, and stroke. Am f Cardiol 1996;77:38-44A

3 The Stroke Prevention in Atrial Fibrillation Investigators. Predictors of thromboembolism in atrial fibrillation. I. Clinical features of patients at risk. Ann Intern Med 1992;116:1-5.

4 Kopecky SL, Gersh BJ, McGoon MD, et al. Lone atrial fibrillation in the elderly. A population-based long term

study [abstract]. Circulation 1989;80(suppl II):409.
5 Abbott W, Maloney RD, McCabe CC, et al. Arterial embolism: a 44 year perspective. Am $\mathcal{F}$ Surg 1982;143:460 4 .

6 Atrial Fibrillation Investigators: AFAAS, BAATAFS, CAFAS, SPAFS and VASPNAFS. Risk factors for stroke and efficacy of antithrombotic therapy in atrial fibrillation. and efficacy of antithrombotic therapy in atrial fibrillation. Analysis of pooled data from five randomiz

7 Rosenqvist M, Lee MA, Moulinier L, et al. Long-term follow-up of patients after transcatheter direct current ablation of the atrioventricular junction. $\mathcal{F} \mathrm{Am}$ Coll Cardiol 1990;16:1467-74.

8 Brignole M, Gianfranchi L, Menozzi C, et al. An assessment of atrioventricular junction ablation and DDDR modeswitching pacemaker versus pharmacological treatment in patients with severely symptomatic paroxysmal atrial fibrillation. A randomized controlled study. Circulation 1997;96: 2617-26.

9 Prystowsky E, Benson DW, Fuster V, et al. Management of patients with atrial fibrillation. A statement for healthcare patients with atrial fibrillation. A statement for healthcare phy and electrophysiology, American Heart Association. phy and electrophysiology, Am

10 Clementy J. La fulguration du fasceau de His. Mise au point à partir de 128 cas personnels consécutifs. Ann Cardiol à partir de 128 cas per
11 Jackman WM, Wang XZ, Friday KJ, et al. Catheter ablation of atrioventricular junction using radiofrequency current in 17 patients. Comparison of standard and large-tip catheter electrodes. Circulation 1991;83:1562-76.

12 Kannel WB, Abbot RD, Savage DD, et al. Epidemiologic features of chronic atrial fibrillation: the Framingham study. N Engl F Med 1982;306:1018-22.

13 Petersen P, Godtfredsen J. Embolic complications in paroxysmal atrial fibrillation. Stroke 1986;17:622-6.

14 Albers GW. Atrial fibrillation and stroke. Arch Intern Med 1994;154:1443-8.

15 Poquet F, Gencel L, Lemetayer P, et al. Long-term survival after closed-chest His-bundle ablation with direct current shock for supraventricular arrhythmias: a 10-year experience with 317 consecutive patients. PACE 1994;17:21505.

16 Jensen SM, Bergfeldt L, Rosenquist M. Long-term follow-up of patients treated by radiofrequency ablation of the atrioventricular junction. PACE 1995;18:1609-14.

17 Canu G, Moncada E, Claudel JP, et al. Evolution à long terme après ablation électrique du fasceau de His dans le traitment des tachycardies supraventriculaires. Arch Mal Coeur 1994;87:1447-51

18 Gasparini M, Lunati M, Magenta G, et al. Reduced embolic risk in paroxysmal and chronic $\mathrm{AF}$ after $\mathrm{AV}$ node ablation [abstract]. Eur 7 Clin Physiol Electrophysiol 1996;6(suppl 5): 81 .

19 Petersen P, Boysen G, Godtfredsen J, et al. Placebocontrolled, randomized trial of warfarin and aspirin for prevention of thromboembolic complication in chronic atrial fibrillation: the Copenhagen AFASAK study. Lancet $1989 ; \mathrm{i}: 175-8$.

20 The Boston Area Anticoagulation Trial for Atrial Fibrillation Investigators. The effect of low dose warfarin on the risk of stroke in patients with nonrheumatic atrial fibrillation. N Engl Med 1990;323:1505-11.

21 Stroke Prevention in Atrial Fibrillation Study Group Investigators. Stroke prevention in atrial fibrillation study: final results. Circulation 1991;84:527-39.

22 Ezekowitz MD, Bridgers SL, James KE, et al, for the Veterans Affairs Stroke Prevention in Nonrheumatic Atrial Fibrillation Investigators. Warfarin in the prevention of Fibrillation Investigators. Warfarin in the prevention of Engl f Med 1992;327:1406-12.

23 Stroke Prevention in Atrial Fibrillation Investigators. Warfarin versus aspirin for prevention of thromboembolism in atrial fibrillation: stroke prevention in atrial fibrillation II Study. Lancet 1994;343:687-91

24 Rodriguez LM, Smeets JLRM, Xie B, et al. Improvement in left ventricular function by ablation of the atrioventricular nodal conduction in selected patients with lone atrial fibrillation. Am 7 Cardiol 1993;72:1137-41.

25 The Stroke Prevention in Atrial Fibrillation Investigators. Predictors of thromboembolism in atrial fibrillation. I. Clinical features of patients at risk. Ann Intern Med 1992;116:6-12

26 Andersen HR, Thuesen L, Bagger JP, et al. Prospective randomised trial of atrial versus ventricular pacing in sick-sinus syndrome. Lancet 1994;344:1523-8.

27 Sgarbossa EB, Pinski SL, Maloney JD, et al. Chronic atrial fibrillation and stroke in paced patients with sick sinus syndrome: relevance of clinical characteristics and pacing modalities. Circulation 1993;88:1045-53. 